\title{
Artritis reumatoidea "juvenil" de forma sistémica en un lactante menor
}

\author{
Patricia Glaser, ${ }^{1}$ Jorge Las Heras, ${ }^{2}$ Anoldo Quezada, ${ }^{3}$ Mario Andreis. ${ }^{3}$
}

\author{
Systemic "Juvenile" rheumatoid arthritis in an infant
}

\begin{abstract}
A classic case of systemic forn of "Juvenile" Rhenmatoid Athritis is described.
The beggining of the disease under six months of age is noted. Treatment with aspirin and high doses of corticosteroids was not succesful]. After a clinical course characterized by a prolonged clinic activity with numerous intercurrent infections the patient died because of a severe varicella complituted with diseminated intravascular coagulation. The characteristics and evolution of this case are discussed.
\end{abstract}

Las afecciones reumatológicas de la infancia están actualmente siendo objeto de progresivo interés en los diferentes centros pediátricos extranjeros. Ello deriva de los mejores métodos de reconocimiento clínico y de laboratorio de las diversas enfennedades del aparato locomotor en el niño. Múltíples disciplinas básicas y clínicas han contribuido al desarrollo del conocimiento patogénico, así como al diseño terapéutico en estas enfermedades.

Entre las artropatías crónicas en el nin̄o, se destaca la Artritis Reumatoidea Juvenil, la cual puede presentarse inicialmente en tres formas clínicas: sistémica, pauciarticular y poliarticular.

La inclusión en uno de estos subtipos esti determinada por las manifestaciones presentes durante los primeros meses de enfermedad. ${ }^{l}$ Por corresponder mejor a la descripción original de StilJ, se tiende a conservar este nombre para la forma sistémica de la Poliartritis Crónica [nfanti] y su frecuencia dentro del total de Artritis Remmatoidea Juvenil oscila alrededor de 10 a $20 \% .^{2,3} \mathrm{La}$ edad de presentación preferente de la Enfermedad de Still varia entre 1 y 5 años, siendo sin embargo posible encontrar casos de ella en niños menores, así como en adultos. ${ }^{+, 5}$ El comienzo en nin̄os menores de un año es más bien raro. ${ }^{6}$

El objeto de esta comunicación es presentar un caso clásico de Enfermedad de Still observado recientemente por los autores, que permite ilustrar $y$ poner de relieve las caracteristicas clínicas

\footnotetext{
I Becario Servicio de Pediatría, Hospital Paula Jaraquemada.

${ }^{2}$ Médico Unidad Patologia Pediattica, Hospital Paulajaraquemada.

${ }^{3}$ Medicos Depto. Meducina Experimental. Laboratorio de Inmunologia, Facultad de Medicinal, División Cięncias Médiçus Sur. Universidad de Chile.
}

de estu forma de Artritis Heumatoidea Juvenil, a la vez que se destaca por haber sido diagnosticado a los 5 meses de edad.

\section{CaSo Clinico}

Niña de 5 meses de edad que ingresó al Servicio de Pediatria del Hospital Paula Jaraquemada por retraso psicomotor atribuido a traumatismo craneocetálico. En el curso de la evolución se nota la presencia de fiebre persistente eindependiente de infecciones intercurrentes. Mientras la sintomatología neurológica se mantiene estacionaria o tiende a resolverse espontíneamente, se hacen evidentes adenopatías axilares bilaterales en forma de racimo, inguinales y cervicales laterales, además de hepatoesplenomegalia y compromiso del estado general (Figuras 1 y 2). Al mes de hospitalización llama la atención el intenso dolor que presenta la paciente al ser movilizada y la posición en semiflexión de las extremidades inferiores; observándose además, aumento de volumen de las articulaciones interfalángicas proximales, ambas rodillas y articulaciones tibio tarsianas, por lo cual se pide una radiogratía de huesos largos que demuestra compromisu de partes blandas periarticulares, rarefacción y disminución de la cortical osea.

Los exámenes del laboratorio mostraron: VHS de 100 mm. Hemograma con 9.400 leucocitos, eosinófilos 1, basófilos 0, mielocitos 1, Juveniles 1, bacilformes 1 , segmentados 17 , linfocitos 72 , monocitos 6, plaquetas nonmales; Factor Reumatoideo negativo. Células de Lupus negativas, C'H50 normal, Electroforesis de proteinas con albúmina de $34,2 \%$, alta 1 globulina $10,4 \%$, al ta 2 globulina $17,1 \%$, betaglobulina $14,2 \%$ y ganaglobulina de $23,8 \%$. El indice albuminaglobulina 


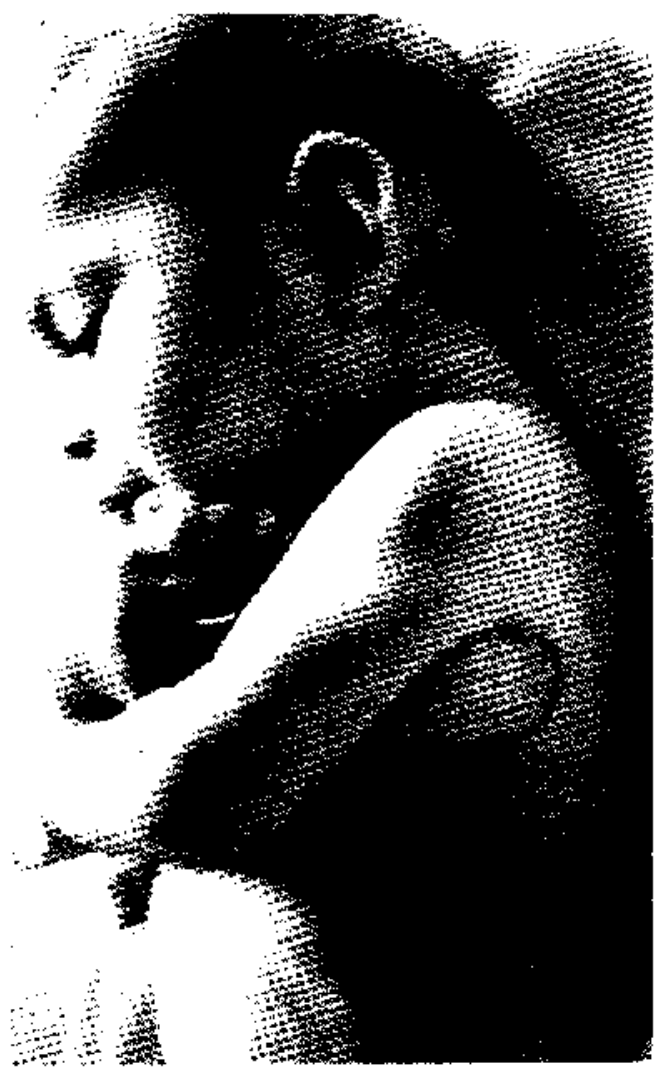

a l. Aumento de volumen de las articulaciones alángicas distales de la mano y las adenopatías es en forma de racinos.

52\%. La radiografia de columna cervical y el ten oftalmológico fúeron nomales.

in el diagnóstico clínico y de laboratorio de tis Reumatoidea Juvenil se comienza el traznto con ácido acetil-salicílico $100 \mathrm{mgs} \times \mathrm{Kg}$ เ paciente evoluciona febril con aumento de nen e intenso dolor de las articulaciones desi. Este compromiso articular fue fluctuante nte toda su enfermedad. Las salicilemias deraron en forma repetida, valores de alrededor mg \%. Estos valores, unidos a la mantención ompromiso general severo, determinaron la ación de $2 \mathrm{mg}$ de prednisona $\mathrm{x} \mathrm{Kg}$. En sidencia con esto se aprecia tendencia a la ralización de la temperatura y progresiva medel estado general, que se vio intemumpida uadros pulmonares intercurrentes que recuin trataniento con antibiotions.

los cinco meses de hospitalización presenta ela que se complica de una neumopatía seven coagulación intravascular diseminada, de fatal.

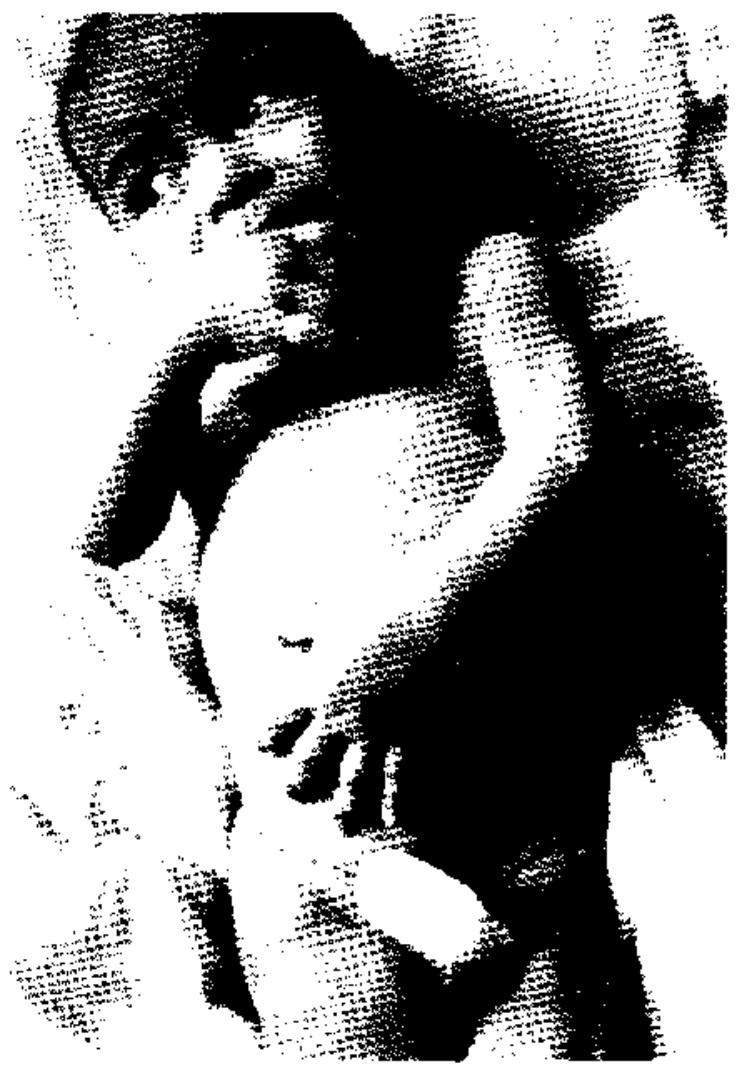

Figura 2. Fascie de angustia, el aumento de volumen de las articulacione's interfialangicas de ambas manos y rodillas y esplenomegaliza.

\section{ANATOMLA PATOLOGICA}

En la piel de ambas piemas, abdomen, tórax y cuero cabelludo se observaron lesiones de varicela en distinto grado de evolución, algunas con signos de infección bacteriana agregada. Microscópicamente estas Iesiones estaban representadas por vesículas epidénicas que contenian líquido, detritus celulares e inclusiones intranucleares.

Ambos pulmones tenian extensos focos de condensación y hemorragia observándose, además, derrame pleural fibrinoso bilateral; microscópicamente en ellos habia marcado infiltrado intersticial, predominantemente mononuclear (monocitos y células plasmáticas), extenso edema intralveolar de tipo fibrinoso y restos de membranas hialinas; no había evidencias claras de inclusiones intracitoplasmáticas o intranucleares. En el corazón se encontró áreas de necrosis y eosinofilia de fibras miocárdicas y en relación con estas áreas, infiltrado mononuclear (células plasmáticas y monocitos). El pericardio mostraba infiltración con 
células mononucleares y depósito de fibrina.

El higado, macroscópicamente, nostraba numerosos focos de necrosis. En los cortes histológicos se observó pérdida de la arquitectura hepática con necrusis hepatocitaria e infiltrado con células mononucleares en los espacios porta. En la periferia de las zonas de necrosis se observaron algunas inclusiones intranucleares. En los riñones se observó extenso compromiso tubular intersticial y glomerular, predontinando la degeneración del epitelio tubular con inclusiones intranucleares ocasionales y el infiltrado monoruclear intersticial. El bazo nostró en la microscopia areas extensas de necrosis con abundantes macrófagos $e$ inclusiones intranucleares ocasionales.

Los ganglios linfáticos eran prominentes cn casi todas las áreas investigadas. Histológicamente estos mostraban moderada a severá depleción de zonas $\Gamma$ y $B$, con reemplazo de los elementos normales por proliferación histiocitaria.

En el estudio de las articulaciones habia engrosamiento y edema en ambas rodillas. Histológicarmente se observó hiperplasia e hipertrofia sinovial con extenso infiltrado inflamatorio crónico, predominantemente perivascular. Este infiltrado estaba compuesto por linfocitos, células plasmáticas e histiocitos (Figura 3 ).

Cubriendo la membrana sinovial se observó abundante fìbrina, parcialmente organizada por proliferación de fibroblastos. La reacción intlamatoria se extendia a la cápsula fibrosa, observándose además, erosión del cartílago subyacente (Figura 4).

\section{DISCUSION}

La paciente presentada corresponde clínicamente a un caso clásico de Artritis Reumatoidea Juvenil de forma sistemica, para la culal se ha reservado especialmente, en la literatura inglesa, el nombre de Enfermedad de Still. La evolución clínica se caracterizó por la fiebre persistente desde un comienzo de sus síntumas. Como en otros casos comunicados, el síndrome febril obligó a descartar una enfennedad infecciosi, e incluso fue, probablemente, la razón para administrar antibióticos en forma empírica. El sindrome febril aislado puede ser la tomna de presentación de la Enfermedad de Still hasta en un $\mathbf{4 5 \%}$ de los pacientes afectados $y$ suele ser catalogado presunti-

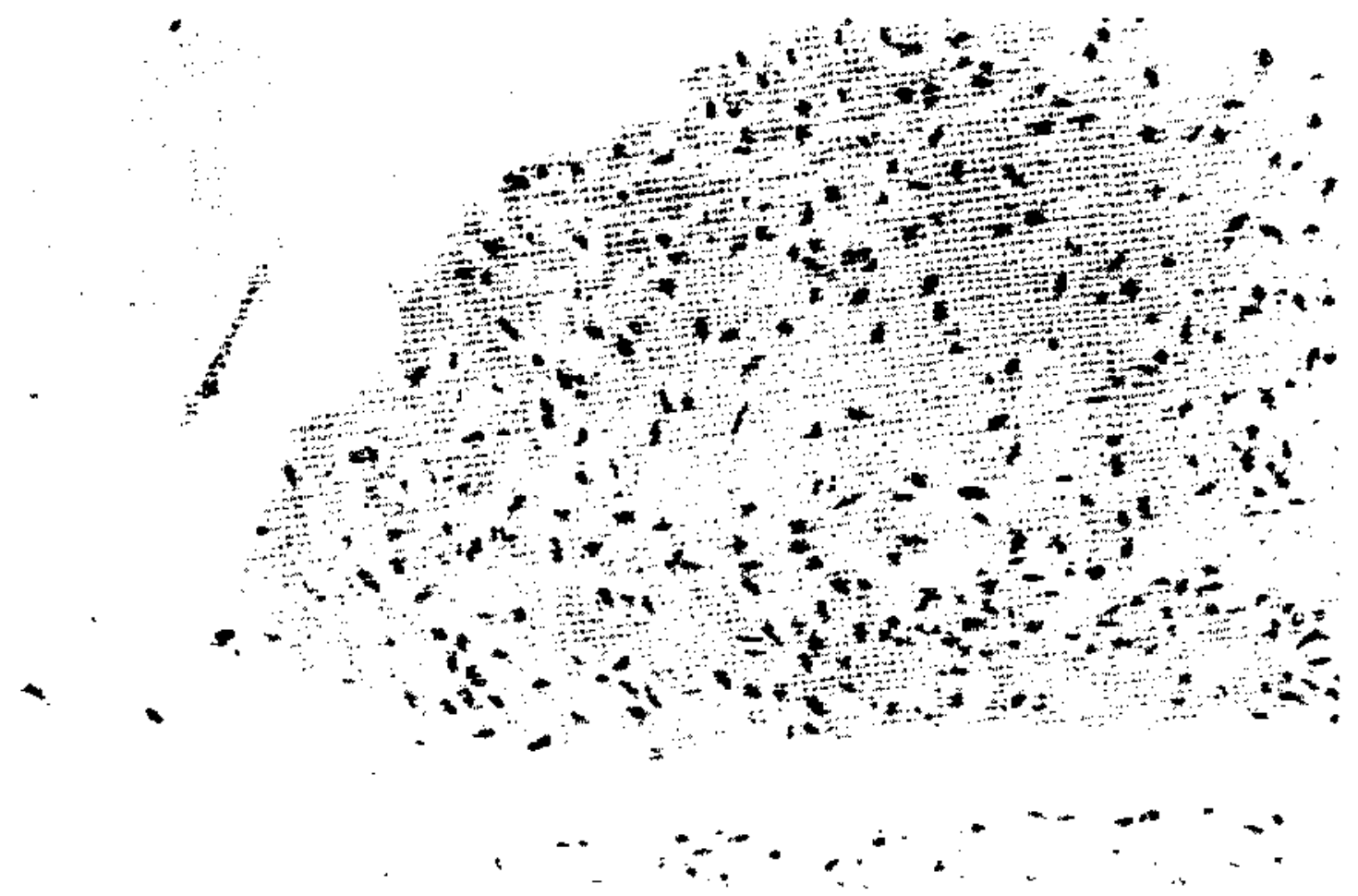

Figura 3. HyE, 40x. Corte histolögioo de membrana sinovial. Marcada hiperplasia e hipertrofia celular, infiltrado intlamatorio eróníco y áreas de necmsis fibrinoide. 


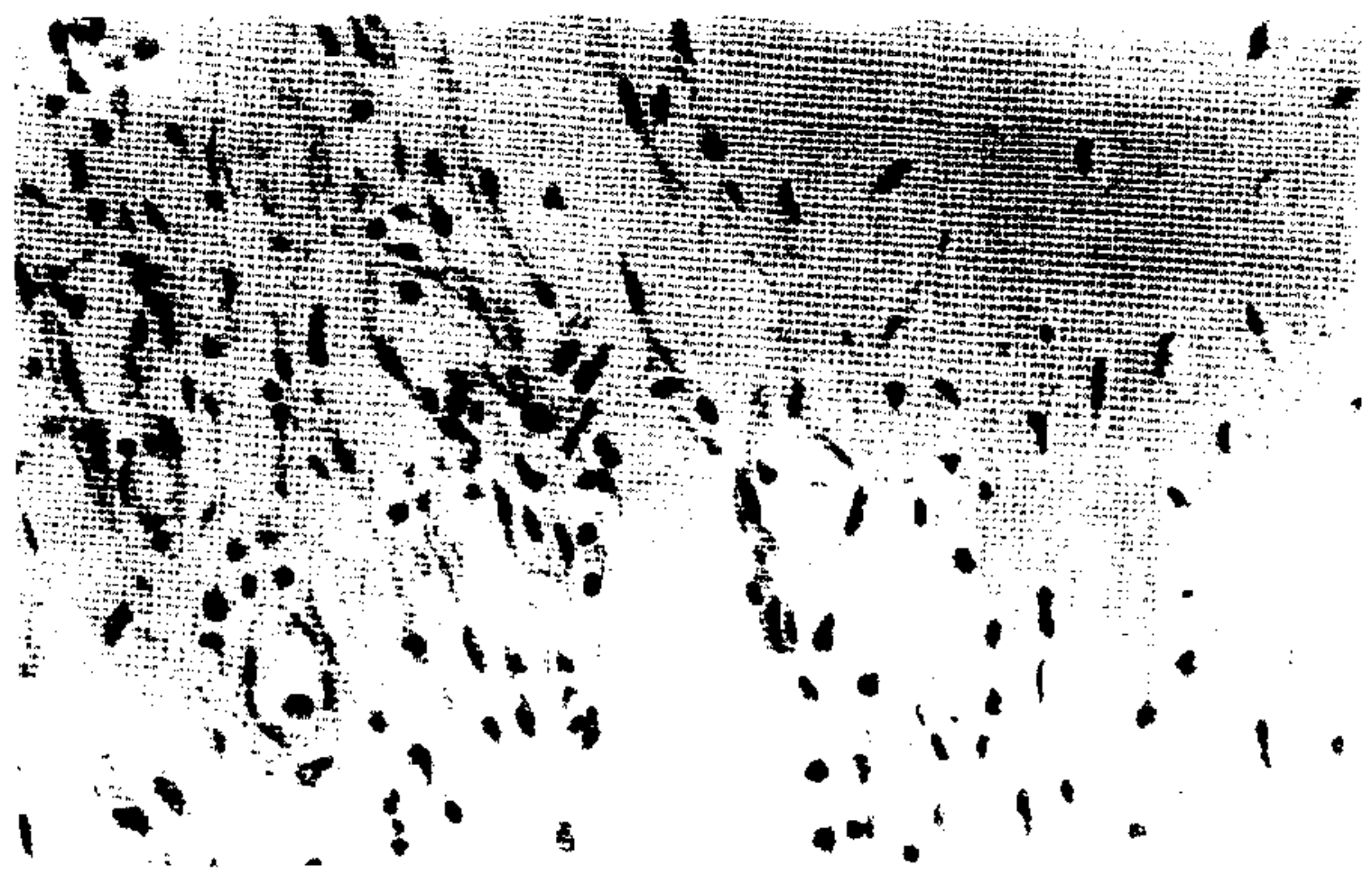

Figura 4. HyE, 40x. Corte histologico de catilago por debajo de la cápsula fibrosa. Ẽrosion e infiltrado inflarnatorio con células plasmáticas y lintócitos.

vamente como fiebre de origen desconocido. ${ }^{i}$

Lo anterior enfatizat la necesiclial de incluir en el diagnósticu diferencial cle la forna sistémicia de Artritis Remnatoidea Juvenil, diversas enternedades intecciosas y la plévade de chadros clinicos que se manitiestan como tiebre de origen obscuro.

La aparición de artritis, riggdez del aparato lucomotor, hepatoesplenomegal ia junto al compromiso general, penuitieron hatcer el diagnóstico e’n nuestra paciente. En esta forma, la paciente completó cinco de low criterios diagnósticos recientemente propuestos pari el diagnóstics de Artritis Reumatoidea Juvenil.' El eritema Inacular característico no estuyo presente en mestra paciente pese a haber sido buscado con las maniolyras adecuadas. Lat explotación del aparato locomotor fue dificil por el dolor $y, \sin$ embargo, la magnitud del cortupromiso indlamatorio hacen muy posible la existencia de tenosinovitis asi como la rigidez axil asociada a la flexión de extremidades prol ablemente traduzca compromiso de las articulaciones de la columna, en particular, del segmento cervical. Ėl estudio serológieo fue negativo en esta paciénte. La presencia de factor remnatoideo en el total de los niños con esta forma de poliartritis crónica es infe- rior al 20\%!' La presencia de anticuerpos antinucleares oscila entro 10 y $40 \%$ de los pacientes. La deteminacion de factor reumatoideo, anticuerpon antinucleares fantinucleoproteínas, antidenxirribonucleico nativo y antiautígeno nnelear extraible) así como cn el antígeno de histocompatibilidad B27, unidos a la observación clinica cuidarlosiz de siguos como el compromiso ocular, lit presencia de núdulos subcutineos y el compromiso preferentc sacroiliaco, penniten scparar a Jos padeientes con Artritis Rematoidea luvenil en cinco grupos con diferente evolucion y pronóstico.'

Vuestra paciente, además de representar un ejemplo clásico de la forna sisténnica tiene el interós de haberse manifestado antes del sexto mes de vida. Eu las publicaciones naciomales, ente 25 casos comurnicados en 1963, sólo se menciona uno, también del sexo femenino, en rque la enfermedad comenzó a los seis meses. Lid evolución fatal de esta enferma coincide con otras comunicaciones en que un cuadro infeccioso tennina con la vida de estos pacientes. Las infecciones como causa de muerte en la Artritis Reumatoidea Juvenil son particulammente frecuentes en aquellos casos en que el fallecimiento es precoz en la evo- 
lución de la enfermedad, antes que aparezca la amiloidosis, que es la otra causa importante de muerte en estos pacientes. La predisposición y gravedad de las infecciones en la Artritis Reumatoidea Juvenil están, seguramente, relacionadas con la naturaleza sistémica de la enfermedad y el trastomo inmunológico subyacente.

La administración de aspirina como base del tratamiento tuvo un resultado muy pobre en nuestra paciente. Controlado con niveles de salicilemias periódicos tue posible demostrar gue el aumento de la aspirina no logró llevar los niveles séricos a cifras adecuadas. No tenemos una explicación clara para los bajos niveles de salicilemia con aporte adecuado de aspirina por vía oral. El deterioro progresivo de la paciente determinó el uso de corticoides con los cuales fue posible apreciar una modesta mejoría cuya evolución fue intermumpida por el brote de varicela que causó el deceso.

El estudio anatomopatológico demostró severas lesiones en prácticamente todos los órganos. Muchas de ellas corresponden, sin lugar a dudas, a la infección varicelatosa y sus consecuencias. Esta complicación hace imposible precisar la magnitud del daño inducido por la enfermed:ad reumatológica.

Este caso pone de manifiesto la necesidad de incluir a la Artritis Reumatoidea Juvenil de forma sistémica, en el diagnóstico diferencial del síndrome febril, con o sin artritis, cuando éste constituye el eje de la presentación clínica, independientemente de la edad de presentación y la eventual concomitancia con síntomas de infección. Así también nos ilustra sobre la gravedad que esta enfermedad puede tener, particularmente cuando en su evolución se injertan cuadros infecciosos diseminados.

\section{RESUMEN}

Se describe un caso clínico clásico de Artritis Reumatoidea Juvenil de forma sistémica. Se destaca la edad de comienzo por debajo de los seis meses de vida. El tratamiento con aspirina y luego dosis altas de corticoides no logró evitar el desenlace fatal de la enfemedad. Luego de un curso caracterizado por actividad clínica mantenida con múltiples infecciones intercurrentes, la paciente falleció de una severa infección varicelatosa complicada de coagulación intravascular. Se discuten las característicus clínicas y la evolución del caso presentado.

\section{REFERENCIAS}

IJRA Criteria Subcommittee of the Diagnostic and Thenapeutic Criteria Committee of the AHA Section of the Arthritis Foundation. Current Proposed Revision of JRA Criteria. Arthritis and Rheumatism (supp.) 20: 195, 1977.

${ }^{2}$ Bytouters, E.G.L.: The History of Pediatric Pheurnatology. Arthritis and Rheumatism (sapp.) 20: 145, 1977.

${ }^{3}$ Schaller, J.G.: Juvenile Fheunatoid Arthritis: Series 1. Arthritis and Rheumatism (supp.) 20: 165, 1977.

${ }^{4}$ Cassidy. J.T.: Juvenile Rheumatoid Anthritis, in: Text-Book of Rlieunatology. EDS.: W.N. Kelley, E.D. Harris, S. Ruddy and C.B. Sledge. W.B. Saunders Co. Pags. 1279-1305, 1981.

${ }^{5}$ Bywaters, E.G.L.: Still's Diserse in the Adult. And. Rheumatic Diseases 30: 121, 1971.

${ }^{6}$ Gadoth, N. and Hershikotitch, Y.: Rheumatoid Arthnitis During the First Year of Life. EUR. J. Pediatr. 132: 115, 1979.

'C Calubro, J.J., Bumstein, S.L. and Staley, H.L. JRA Posing as Fever of Unknown Orign. Anthritis and Bheumatism (isupp.) 20: 178, 1977 .

"Gomez, M. and Andreis, M.: Clinical Aspects of Juvenile Rheuriatoid Arthritis. Arch Interamer. Hheumatology 6: 224, 1963.

${ }^{4}$ Batm, J, and Gutotoskat, G.: Death in Juvenile Rheumatoid Arthritis. Arthritis and Pheumatism (Supp.) 20: 253, 1977. 